\title{
Evaluation of roselle based on the internal transcribed spacer sequence of rRNA gene
}

\author{
Se Hee Park', Florence C. Ginibun², Mark S. Roh ${ }^{3}$, Ming-Xi Li ${ }^{3}$, Jeung Keun Suh ${ }^{3}$, Young Hee Joung ${ }^{1 *}$ \\ ${ }^{1}$ Chonnam National University, School of Biological Sciences \& Technology, Gwangju, Korea; ${ }^{2}$ University of Malaya, Faculty of \\ Science, Institute of Biological Sciences, Genetics and Molecular Biology Unit, Kuala Lumpur, Malaysia; ${ }^{3}$ Dankook University, School of \\ Bio-Resource Science, Laboratory of Floriculture and Plant Physiology, Cheonan, Chungnam, Korea
}

\section{A B S T R A C T}

Genetic differences in 5-lobed leaf Roselle (Hibiscus sabdariffa L.) collected from China, Malaysia, Sudan, and USA were evaluated by the sequence of nuclear internal transcribed spacer 1, 2 regions in rRNA gene (ITS 1, 2 regions). A total of 27 samples were subjected to polymerization chain reaction (PCR)and 729 bp bands were amplified in all samples. Samples were divided into 4 groups based on the single nucleotide polymorphisms at 71, 91, and 249 of the 729 bp band. However, no variations among samples collected from different geographic origins of $H$. sabdariffa are observed.

Keywords: Hibiscus sabdariffa; ITS 1; 2 regions; Single nucleotide polymorphism (SNP)

\section{INTRODUCTION}

Hibiscus sabdariffa, commonly known as Roselle, is produced for tea from the brilliant red sepals or calyces because they have a rich content of vitamins A, C, and several antioxidants (USDA, 2014). The best quality Roselle is produced in the Sudan and China, but Egypt, Jamaica, Mexico, and USA are also important suppliers.

Characterization and identification of genetic difference within the species/varieties is very important for utilization and conservation of Roselle resources. So several methods were performed such as randomly amplified polymorphic DNA (RAPD), inter simple sequence repeats (ISSR) and inverse sequence-tagged repeat (ISTR) to characterize Roselle (Torres-Moran et al., 2011; Khafaga, 2013; Omalsaad et al., 2014). Hibiscus sabdariffa with 5-lobed and a mostly red calyx whereas $H$. cannabinus accessions with 3 -lobed leaves and mostly a yellow calyx were separated using RAPD markers, but separation of some accessions in the same species were not successful (Omalsaad et al., 2014). H. sabdariffa cultivars collected from different locations and showed three different phenotypes were divided only two group using RAPD and ISSR marker (Khafaga, 2013). Hibiscus sabdariffa accessions with 3-lobed leaves were separated from those with 5-lobed leaves using ISTR markers and it was concluded that accessions collected from China and other areas probably share a common origin among the twelve genotypes grown in western Mexico (Torres-Moran et al., 2011).

From these reports, RAPD, ISSR and ISTR generated useful molecular markers for identification but it was not successful to reveal the genetic difference within closely related H. sabdariffa as. Markers generated from the ITS 1, 2 regions of nuclear rRNA gene are considered as useful to study closely related species (Baldwin, 1992). SNPs based on the sequence information of the ITS 1, 2 regions were used in identification of genetic relationships among seedlings of Kolkwitzia (Park et al., 2014) and Ligustrum (Ma et al., 2014). The objective of this experiment was to understand the genetic relationships of $H$. sabdariffa $\mathrm{L}$. collected from 4 different countries using SNPs of the ITS 1, 2 regions.

\section{MATERIALS AND METHODS}

Roselle (Hibiscus sabdariffa L.) seeds were collected from China, Malaysia, USA (purchased from the Seedville), and Sudan. The germinated seeds were grown at greenhouse and transplanted into 5-gallon pots after one year.

\footnotetext{
${ }^{*}$ Corresponding author:

Y. H. Joung, Chonnam National University, School of Biological Sciences \& Technology, Gwangju, Korea. E-mail: yhjoung@chonnam.ac.kr

Received: 11 January 2015;

Revised: 13 April 2015;

Accepted: 28 April 2015;

Published Online: 04 May 2015
} 
A total of 27 Roselle plants with 5-lobed leaves; 4 from Sudan, 4 from USA, 4 from Malaysia, and 15 from China were randomly selected from 30 plants from each origin. Twenty four weeks after transplanting, young and expanding leaves were collected for extraction of total genomic DNA using SolgTM Genomic DNA prep kit (SolGent, Daejeon, Korea). To amplify the ITS 1, 2 regions, PCR was performed with a $18 \mathrm{~S}$ rRNA gene specific forward primer ITS 1 (5'- TCC GTA GGT GAA CCT GCG G -3') and a $28 \mathrm{~S}$ rRNA gene specific reverse primer ITS 4 (5'- TCC TCC GCT TAT TGA TAT GC -3'). The PCR reaction mix contained template DNA (20 ng), each primers (10 pmols), $2.5 \mu$ l of $10 \times$ PCR buffer and $0.15 \mu \mathrm{l}$ of Pfu DNA Polymerase (SolGent). The PCR amplification condition was: $2 \mathrm{~min}$ at $95^{\circ} \mathrm{C}$, followed by 35 cycles of $20 \mathrm{~s}$ at $95^{\circ} \mathrm{C}, 40 \mathrm{~s}$ at $56^{\circ} \mathrm{C}$, and $1 \mathrm{~min}$ at $72^{\circ} \mathrm{C}$. The PCR products were purified with a gel purification kit (SolGent) according to the manufacturer's direction and direct sequenced. Sequences were compared following the BLAST search at NCBI. (http://blast.ncbi.nlm.nih.gov/).

\section{RESULTS AND DISCUSSION}

Leaves of all samples collected from 4 countries had palmate 5-lobed leaves with a slight variation in morphology of leaflets (Fig. 1). The shape and color of the calyx showed no significant visual differences among samples collected from each country.

After PCR, 729 bp products were amplified from all samples and $581 \mathrm{bp}$ products were amplified from some samples (Fig. 2). When the 729 bp PCR bands were sequenced, double peaks were observed in 3 SNP positions at 71, 95, 249 (Fig. 3) with ambiguities following International Union of Pure and Applied Chemistry (IUPAC) and nucleotide ambiguity codes revealed 4 different types. The

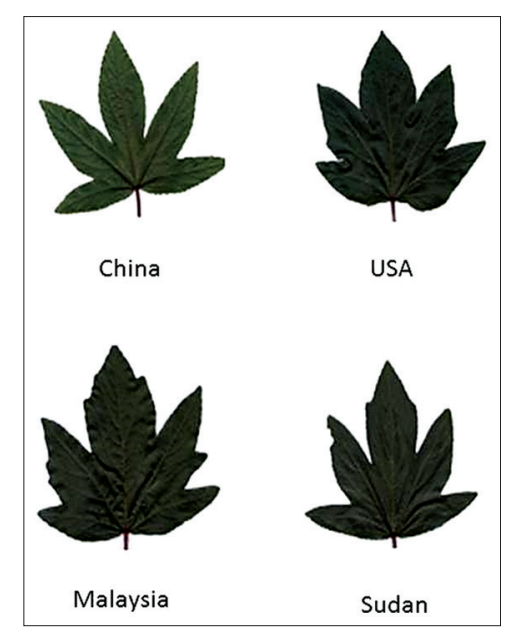

Fig 1. General leaf morphology with 5 lobes collected from China, Malaysia, USA and Sudan.
A type with IUPAC code $\mathrm{R}$ in position 249 were observed from 11 samples collected from China and 2 samples from USA (U05, U07), B type with code $\mathrm{Y}$ in position 95 in one sample collected from USA (U06), and two samples from Malaysia (M14, M15), C type with code $\mathrm{R}$ in position 249 and code $\mathrm{Y}$ in position 95 from two samples from China (C05, C11) and one from Malaysia (M16), and D type with code $\mathrm{W}$ in position 71 observed in two samples from Sudan (S02, S03) and one from Malaysia (M13).

If the nucleotide of ambiguity codes are matched with the code of the remaining samples, for example code $\mathrm{W}$ to nucleotide $\mathrm{A}$ at position 71 , no variances in the nucleotide sequences of ITS 1, 2 regions from two from Sudan and one from China and among all samples are observed. Therefore, there are no variations among all samples collected from different origins based on the ITS 1, 2 regions haplotypes as reported for accessions grown in Mexico (Torres-Moran et al., 2011).

The 729 bp sequences were blasted on NCBI BLAST, and all showed over 99\% identity with Hibiscus sabdariffa var. sabdariffa (FJ527608.1) and H. sabdariffa var. altissima (JQ609255.1). This suggests that H. sabdraiffa and H. cannabinus cannot be distinguished using SNPs, although they were separated using RAPD markers (Omalsaad et al., 2014). Sequences of ITS 1, 2 regions of one sample each of four types were registered at NCBI GenBank: U05 (A type, KP262031), U06 (B type, KP262033), M16 (C type, KP262032), and S02 (D type, KP262034).

The 581 bp PCR bands were also amplified with 729 bp band from China (C13), Sudan (S03) and Malaysia sample (M15). These $581 \mathrm{bp}$ were isolated and sequenced. The sequences of $581 \mathrm{bp}$ DNA were matched with rRNA ITS 1,2 of Aureobasidium species (KF887041, JN031005).

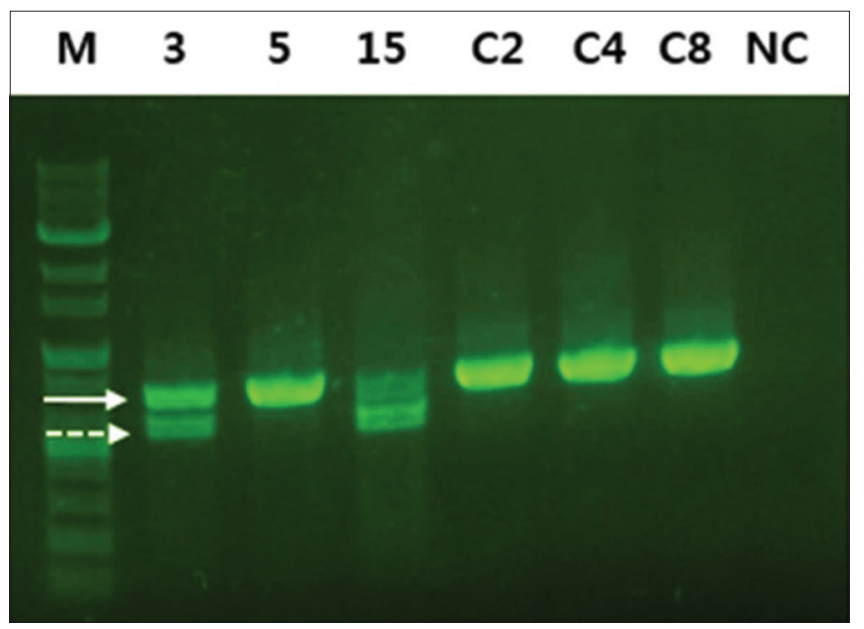

Fig 2. PCR analysis using the ITS1, 2 primers in Hibiscus leaves showing one large band of $729 \mathrm{bp}$ (solid line) and small band of $581 \mathrm{bp}$ (dashed arrow). M: DNA ladder and NC: negative control. 


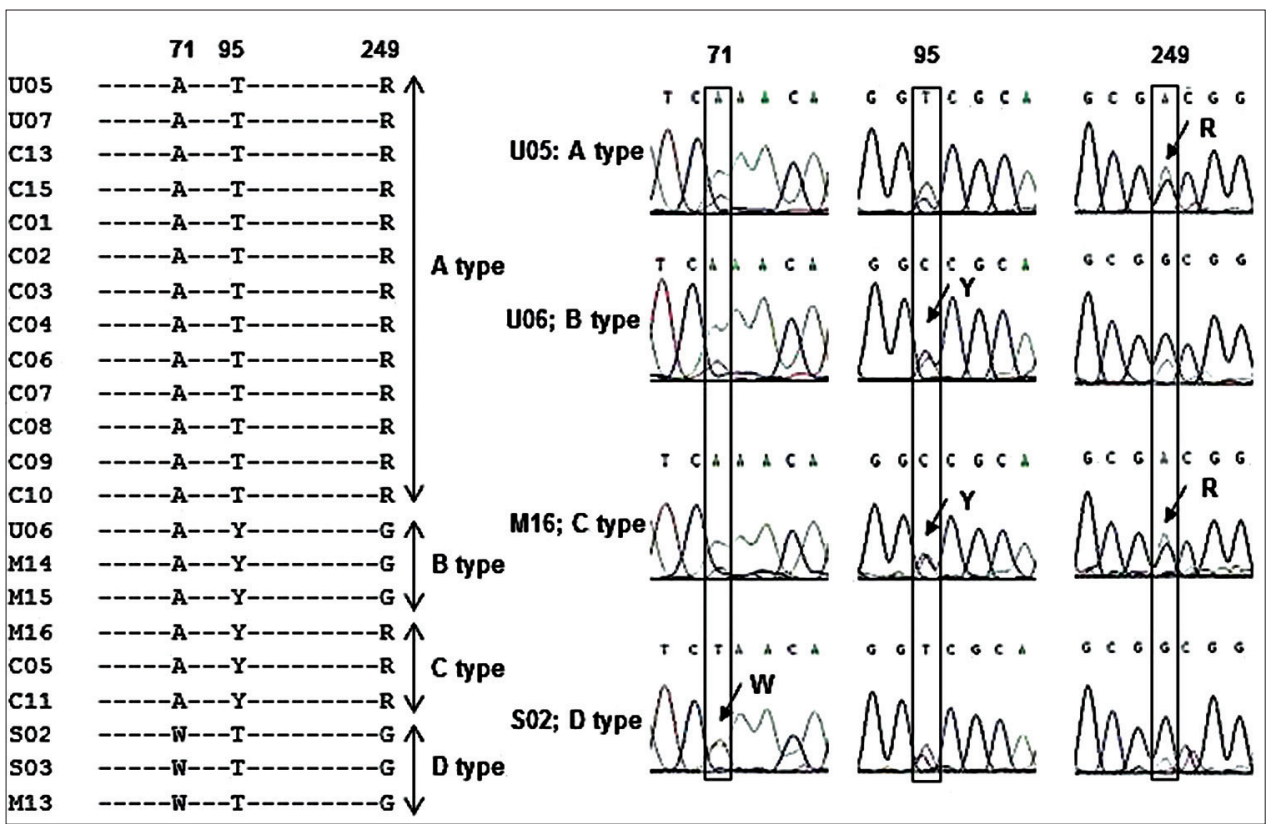

Fig 3. A portion of sequences of ITS nrDNA region and sequence chromatograms of Hibiscus samples from China (C01 - C15), Malaysia (M13 - M16), USA (U505 - U07), and Sudan (S02 - S03) are presented. (A) Four different types, A, B, C, and D type with sample numbers and a portion of nucleotide sequence with IUPAC ambiguity codes at the position 71, 95, and 249 are presented. (B) Sequence chromatograms showing two peaks at three sites. Arrows indicated IUPAC ambiguity codes (W: A or T, Y: C or T, R: A or G).

This result suggest that these samples may be contaminated with fungus Aureobasidium species which is naturally occurring as epiphyte or endophyte to a wide range of plant species (Andrews et al., 2002).

\section{CONCLUSION}

Hibiscus sabdariffa collected from China, Malaysia, Sudan, and USA were evaluated by the ITS 1, 2 regions and they were divided into 4 groups based on the sequence. However, variations among samples collected from different geographic origins were not observed.

\section{ACKNOWLEDGMENTS}

We thank Drs. D. Lakshman and R. G. Anderson for their comments and editing to improve this manuscript. Seeds were kindly provided from Mohamed Omer Elsheikh, Faculty of Animal Production, University of Gezira, Sudan and from the Department of Agriculture, Malaysia.

\section{Author contributions}

Y. H. Joung contributed to all aspects of this manuscript. S. H. Park was involved in the DNA research work. M-X. $\mathrm{Li}$ and J. K. Suh were involved in the phenotype analysis. F. C. Ginibun and M. S. Roh helped in sample collection and editing of the manuscript.

\section{REFERENCES}

Andrews, J. H., R. N. Spear and E. V. Nordheim. 2002. Population biology of Aureobasidium pullulans on apple leaf surfaces. Can. J. Microbiol. 48: 500-513.

Baldwin, B. G. 1992. Phylogenetic utility of the internal transcribed spacers of nuclear ribosomal DNA in plants: An example from the compositae. Mol. Phylogenet. Evol. 1: 1-16.

Khafaga, A. F. A. 2013. Molecular genetic identification of some Egyptian hibiscus samples. J. Am. Sci. 9: 28-35.

Ma S. H., G. Burchi, J. K. Suh, M. S. Roh and Y. H. Joung. 2014. Identification of Ligustrum seedlings based on sequence analysis of an internal transcribed spacer. Hort. Environ. Biotechnol. 55: 423-427.

Omalsaad, A. K., M. A. Islam, M. A. Jahan, Z. Yaakob and M. Osman. 2014. Genetic relationship between roselle (Hibiscus sabdariffa L.) and kenaf (Hibiscus cannabinus L.) samples through optimization of PCR based RAPD method. Emirates $\mathrm{J}$. Food Agric. 26: 247-258.

Park S. H., G. Burchi, M. S. Roh and Y. H. Joung. 2014. Characterization of Kolkwitzia amabilis accessions based on flowering and molecular marker. Sci. Hort. 165: 190-195.

Torres-Moran M. I., M. Escoto-Delgadillo, J. Ron-Parra, G. Tarra-Tovar, S. Mena-Munguia, A. Rodriguez-Garcia, A. Rodriguez-Sahagun and $O$. Castellanos-Hernandez. 2011. Relationship among twelve genotypes of roselle (Hibiscus sabdariffa L.) cultivated in western Mexico. Ind. Crops Prod. 34: 1079-1083.

US Department of Agriculture, Agricultural Research Service, National Nutrient Database for Standard Reference, Release 27. 2014. Available from: http://www.ndb.nal.usda.gov/ndb/ foods. [Last accessed on 2014 Jan 15]. 